\title{
Reversed and anomalous Doppler effects in photonic crystals and other time-dependent periodic media
}

\author{
EVAN J. REED ${ }^{\mathrm{a}, \mathrm{b}, *}$, MARIN SOLJAČIĆ ${ }^{\mathrm{a}}$, MIHAI IBANESCU ${ }^{\mathrm{a}}$ and \\ J. D. JOANNOPOULOS \\ a Department of Physics, Massachusetts Institute of Technology, Cambridge, MA 02139, USA \\ ${ }^{\mathrm{b}}$ Lawrence Livermore National Laboratory, Livermore, CA 94550, USA
}

Received 10 April 2005; Accepted 19 May 2005

\begin{abstract}
We predict that reversed and anomalous non-relativistic Doppler shifts can be observed under some circumstances when light reflects from a shock wave front propagating through a photonic crystal, or material with a periodic modulation of the dielectric. This theoretical prediction is generalizable and applies to wave-like excitations in a variety of periodic media. An experimental observation of this effect has recently been made (Seddon, N. and Bearpark, T., Science, 302 (2003) 1537) and we provide a brief discussion of this experiment.
\end{abstract}

Keywords: Doppler effect, photonic crystal, shock wave

\section{Introduction}

Photonic crystals [1-4], or periodic dielectric media, have received much recent attention as a way to control the propagation of electromagnetic radiation on length scales comparable to the wavelength of the radiation. This paper focuses specifically on the dielectric mirror or "multi-layer stack" type of photonic crystal consisting of a 1D structure of alternating layers of different dielectric materials. While each individual dielectric material comprising the dielectric mirror transmits light, light of certain frequencies incident on the mirror structure normal to the layers will be completely reflected if the wavelength falls within specific ranges. The reason is that the light is scattered at the layer interfaces, and if the layer spacing is chosen appropriately, the multiply-scattered waves can interfere destructively inside the material to prevent transmission. An analogy of this phenomenon can be drawn to Bragg reflection of electrons from a crystal lattice.

Theoretical studies of photonic crystals are often performed by solving Maxwell's equations using a finite-difference computational approach, commonly referred to as finite-difference time-domain (FDTD) simulations (see, e.g. Ref. 5). This approach solves Maxwell's equations exactly except for the discretization of time and space. Calculation of the propagation of radiation in a photonic crystal is simplified considerably compared to calculation of the propagation of electrons in an atomic crystal since photons are non-interacting (unless the intensity is strong enough to induce

*To whom correspondence should be addressed, E-mail: reed23@llnl.gov 


\section{Evan J. Reed et al.}

non-linearities in the dielectric.) For this reason, electromagnetic properties of photonic crystals can be calculated to a higher degree of accuracy (essentially exactly) than electronic properties of atomic crystals.

The properties of photonic crystal structures can be altered using a wide variety of means including liquid-crystal effects [6], free-carrier injection [7], and optical Kerr effects [8]. These effects have been considered in approaches to yield reconfigurable or tunable photonic crystal structures, but the effects of light interacting with such structures while the photonic crystal undergoes reconfiguration have received little attention until recently [9-13]. Toward this end, we have recently performed computer simulations and analytical theory that demonstrate that novel and unexpected phenomena can occur when light interacts with a shock wave or shock-like modulation of the dielectric propagating through a photonic crystal $[14,15]$. A physical, compressive shock wave can produce large changes in dielectric properties of materials on 1-1000 picosecond timescales. Alternatively, a shock-like modulation of the dielectric of a photonic crystal can be produced using non-linear or other approaches.

One of the effects we have predicted to occur when light reflects from a shock wave propagating through a photonic crystal is a frequency shift of light across the photonic crystal bandgap. This effect can be interpreted as an anomalously large Doppler shift with frequency change 3-4 orders of magnitude larger than the usual Doppler shift that might be expected when light reflects from an object moving at the shock propagation speed. The frequency shift is endowed upon light that is trapped in a cavity at the shock front. Frequency shifting occurs as a result of shock compression of the cavity while light is trapped inside. Another predicted effect is a bandwidth narrowing effect whereby the bandwidth of electromagnetic radiation can be compressed by a factor of four or more while preserving up to $100 \%$ of the energy of the radiation.

A third prediction of our recent work is that a reversed Doppler effect can be observed when light reflects from a shock wave propagating through a photonic crystal [15]. This prediction is the subject of this paper. Under some circumstances, light can be shown to reflect with frequency shift opposite in sign to that expected for the usual Doppler shift. This frequency shift can also have unusual magnitudes. Recently, this theoretical prediction was confirmed by an experimental observation of a reversed Doppler shift from a shock-like wave propagating through a periodic electrical transmission line [1]. While the experiment by Seddon and Bearpark was performed in an electrical transmission line rather than a photonic crystal, the physical origin of the effect in these two systems is closely related.

Until our recent work, non-relativistic reversed Doppler shifts had only been predicted to occur in pathological systems with simultaneously negative effective permittivity and permeability [16,17]. Anomalous Doppler effects have been observed in plasmas that propagate at near-relativistic speeds [18]. The anomalous Doppler effects presented in this paper have a fundamentally different physical origin than in both of these systems and can be observed at non-relativistic speeds in systems with linear optical materials. In the shocked photonic crystal system, up to $100 \%$ of the incident light energy can be transferred into the anomalous Doppler shift.

In this paper, we first present theoretical predictions of reversed Doppler effects that occur when light reflects from a shock wave propagating through a photonic crystal. This is followed by a discussion of the first experimental bservation of this 
effect by Seddon and Bearpark in an electrical transmission line and a discussion of the connection to our theoretical prediction.

\section{Theoretical prediction of Anomalous Doppler effects in shocked photonic crystals}

The Doppler effect predicts that light shined by an observer onto an object moving toward him will be reflected with a higher frequency. In this section, we show that the usual theory behind the Doppler shift breaks down for light reflected from a shock wave propagating in a photonic crystal. We employ detailed numerical simulations and analytical theory to prove that anomalous Doppler shifts, both in sign and magnitude, can be observed.

\subsection{Computational EXPERiments}

In this section, we perform computational experiments on the interaction of electromagnetic radiation with a model of a shock wave in a photonic crystal by numerically time-evolving Maxwell's equations using an FDTD scheme in 1D with a single polarization of the electric and magnetic fields,

$$
\begin{aligned}
& \frac{\partial E}{\partial x}=-\frac{1}{c} \frac{\partial B}{\partial t} \\
& \frac{\partial B}{\partial x}=-\frac{1}{c} \frac{\partial(\epsilon(x, t) E)}{\partial t}
\end{aligned}
$$

The time and space-dependent dielectric function $\epsilon(x, t)$ represents the shocked photonic crystal.

A model $\epsilon(x, t)$ for the effects of a shock wave propagating in the 1D photonic crystal considered in this section is shown in Figure 1. The pre-shocked crystal (on the right) is comprised of two materials with identical bulk moduli (and therefore identical sound speeds) but differing dielectric. One layer has length $d_{1}=0.2 a$ and the other has length $d_{1}=0.8 a$, where $a$ is the pre-shock lattice constant. The compression

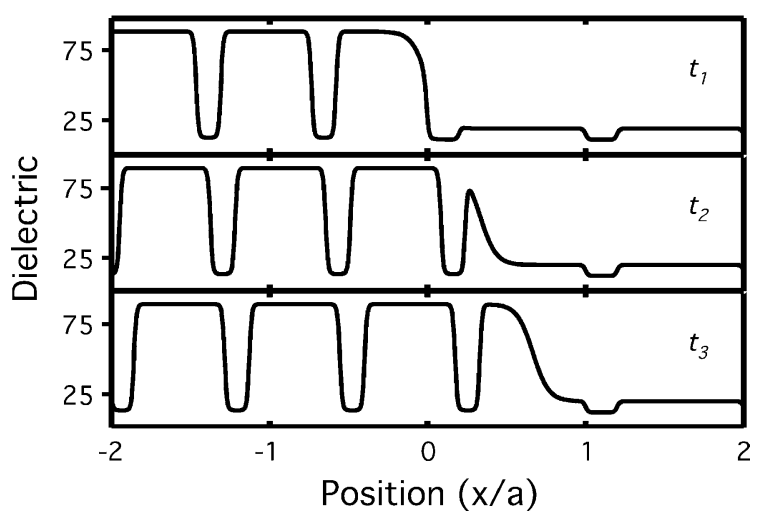

Figure 1. Dielectric as a function of position for three equally-spaced instants in time, $t_{1}<t_{2}<t_{3}$. The shock front moves at a constant velocity, and the material behind the shock moves at a smaller constant velocity. For this model, the dielectric ranges from 2.1 to 11.0 before the shock front and 3.7 to 89.4 behind the shock front. These large values are for computational tractability only. All the results of this work can be observed with physical values as discussed in the text. 
of the lattice by the shock wave has two key effects on the photonic crystal: the lattice is compressed and the dielectric is changed through a strain dependence. If we focus on materials where the dielectric constant is increased with compression, these two main effects affect the bandgap frequency in opposing ways in the shock-compressed material: decrease of the lattice constant increases the bandgap frequency, but increasing the dielectric lowers the bandgap frequency. The bandgap can be made to decrease in frequency upon compression if materials with a sufficiently large dielectric dependence on strain are employed, $\frac{d \epsilon}{d s}$, where material strain is given by $s$. Materials that are used for acousto-optical modulation in particular can have a large negative dielectric dependence on strain [19]. As an illustrative example, we have chosen parameters for our model system with $\frac{1}{\epsilon_{1}} \frac{d \epsilon_{1}}{d s_{1}}=-2.9$ and $\frac{1}{\epsilon_{2}} \frac{d \epsilon_{2}}{d s_{2}}=-26.5$, a bulkmodulus for both materials of $37 \mathrm{GPa}$, and a shock pressure of $10 \mathrm{GPa}$. The strain dependence of the dielectric is exaggerated purely due to the computational requirement of very long simulations and fine spatial discretization for realistic parameters. All effects proposed in this section can be observed in experimentally accessible scenarios. For example, a photonic crystal with a bandgap width of $10^{-2} \omega_{\text {gap }}$ made of large layers of tellurium and small layers of another material shocked to a strain of around $1 \%$ will produce frequency shifts of $3 \times 10^{-7} \omega$ which are readily observable experimentally.

The time-dependent 1D dielectric shown in Figure 1 is composed of bilayer regions (periodic unit cell consists of two different dielectric materials) where the location of the interfaces between bilayers in the shocked crystal $\left(x_{1, j}(\hat{t}), x_{2, j}(\hat{t})\right)$ (in units of the pre-shocked lattice constant $a$ ) are given in terms of the locations of the interfaces between bilayer regions in the unshocked crystal $\left(\tilde{x}_{1, j}, \tilde{x}_{2, j}\right)$ as,

$$
x_{i, j}(\hat{t})=\tilde{x}_{i, j}-\frac{p}{2 B}\left(\tilde{x}_{i, j}-v \hat{t}\right)\left(\tanh \left(-\gamma\left(\tilde{x}_{i, j}-v \hat{t}\right)\right)+1\right) .
$$

The shock speed is $v$, the shock front thickness is given by $\gamma^{-1}$, the final shock pressure by $p$, and the bulk modulus for both materials by $B$. The time has units of $\hat{t} \equiv a t / c$. The variation of $\epsilon\left(\hat{x} \equiv \frac{x}{a}\right)$ in the shocked crystal is given in terms of the bilayer interfaces as (neglecting the strain dependence of $\epsilon(\hat{x})$ ),

$$
\begin{aligned}
& \epsilon(\hat{x}, \hat{t})=\frac{1}{2}\left(\epsilon_{1}+\epsilon_{2}\right)+\frac{1}{2}\left(\epsilon_{2}-\epsilon_{1}\right) \tanh \left(\delta\left(\hat{x}-x_{1, j}\right)\right), \frac{1}{2}\left(x_{1, j}+x_{2, j-1}\right) \leq \hat{x}<\frac{1}{2}\left(x_{2, j}+x_{1, j}\right) \\
& \epsilon(\hat{x}, \hat{t})=\frac{1}{2}\left(\epsilon_{1}+\epsilon_{2}\right)+\frac{1}{2}\left(\epsilon_{2}-\epsilon_{1}\right) \tanh \left(-\delta\left(\hat{x}-x_{2, j}\right)\right), \frac{1}{2}\left(x_{2, j}+x_{1, j}\right) \leq \hat{x}<\frac{1}{2}\left(x_{1, j+1}+x_{2, j}\right) .
\end{aligned}
$$

The time-dependence of $\epsilon$ enters from the time-dependence of the dielectric region interfaces, $\left(x_{1, j}(\hat{t}), x_{2, j}(\hat{t})\right)$. The dielectric alternates between $\epsilon_{1}$ and $\epsilon_{2}$ with tanh splines of width $\delta^{-1}$ between regions to prevent a moving discontinuity. To account for the strain dependence of the two dielectric regions, we apply a transform to the dielectric given by Eq. (3),

$$
\epsilon(\hat{x}, \hat{t}) \rightarrow \epsilon(\hat{x}, \hat{t})\left[1+\left(\frac{1}{\epsilon_{1}} \frac{d \epsilon_{1}}{d s_{1}}+\left(\frac{1}{\epsilon_{2}} \frac{d \epsilon_{2}}{d s_{2}}-\frac{1}{\epsilon_{1}} \frac{d \epsilon_{1}}{d s_{1}}\right) \frac{\left(\epsilon(\hat{x}, \hat{t})-\epsilon_{1}\right)}{\left(\epsilon_{2}-\epsilon_{1}\right)}\right) \frac{p}{2 B}(\tanh (-\gamma(\hat{x}-v \hat{t}))+1)\right] .
$$

In Figure 1, the thickness of the shock wave front $\left(\gamma^{-1}\right)$ is $1, \delta^{-1}=\frac{1}{60}$. Figure 2 is a schematic of the bandgap frequencies in front of and behind the shock front for the dielectric given in Figure 1. The 1st bandgap is lowered in frequency as the shock 


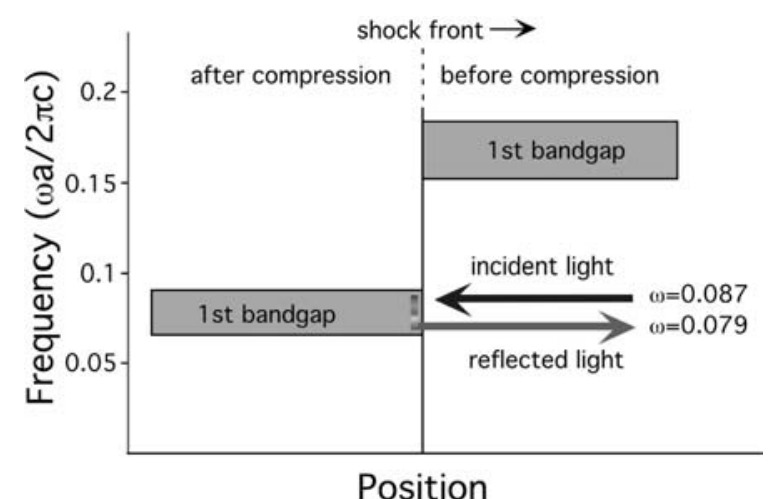

Figure 2. Schematic of a shock wave moving to the right that compresses the lattice but lowers the bandgap frequency due to a strain dependence of the dielectric. Light incident from the right reflects from the post-shock bandgap with a reversed Doppler shift.

compresses the photonic crystal. Consider now continuous-wave electromagnetic radiation incident from the right (opposite to the direction of shock propagation) with frequency within the 1st bandgap of the post-shock crystal as shown in Figure 2. The frequency of this radiation is far from the 1st bandgap edge in the pre-shock crystal. The incident light is reflected and acquires a reversed Doppler shift, i.e. lowered frequency in this case.

Figure 3 shows the magnetic field for a FDTD simulation where this reversed Doppler effect is observed. The shock front (dashed line) has thickness $\gamma^{-1}=2$ and propagates to the right with $v=1.5 \times 10^{-2} c$, which is chosen to be artificially high for computational considerations. The panels in Figure 3 are obtained by Fourier transforming the magnetic field over windows of time $(\Delta t=500 \mathrm{a} / \mathrm{c})$ beginning at the times shown in the upper right corners. This window duration is long enough to distinguish between the incident and reflected frequencies, but short enough to distinguish between different times during the simulation. The top panel shows light incident from a source on the right, and the bottom panel shows this light reflecting with a decreased frequency.

Figure 4 shows a similar FDTD simulation where the shock front is considerably sharper, $\gamma^{-1}=0.1$. Here, $v=3 \times 10^{-3} c$ and the Fourier transform is performed over a time $(\Delta t=3500 a / c)$. The criteria for choice of this window are the same as those discussed in Figure 3. Multiple, equally-spaced reflected frequencies are observed in this case. These frequencies are a result of the time-periodic nature of the shock propagation over a periodic structure. The interpretation of the reflected light as multiple equally-spaced frequencies or a temporally periodic modulation of a single carrier frequency depends on the window length utilized for the Fourier transform and is therefore a matter of resolution in the experimental detection apparatus. For a photonic crystal designed to reflect light of $1 \mu \mathrm{m}$ wavelength and with a shock speed of $10 \mathrm{~km} / \mathrm{s}$, the periodic modulation typically has a frequency on the order of $10 \mathrm{GHz}$.

\subsection{AnAlysis}

The key reason for the observation of an anomalous Doppler shift from the shock front can be discovered by careful consideration of the origin of the usual Doppler 


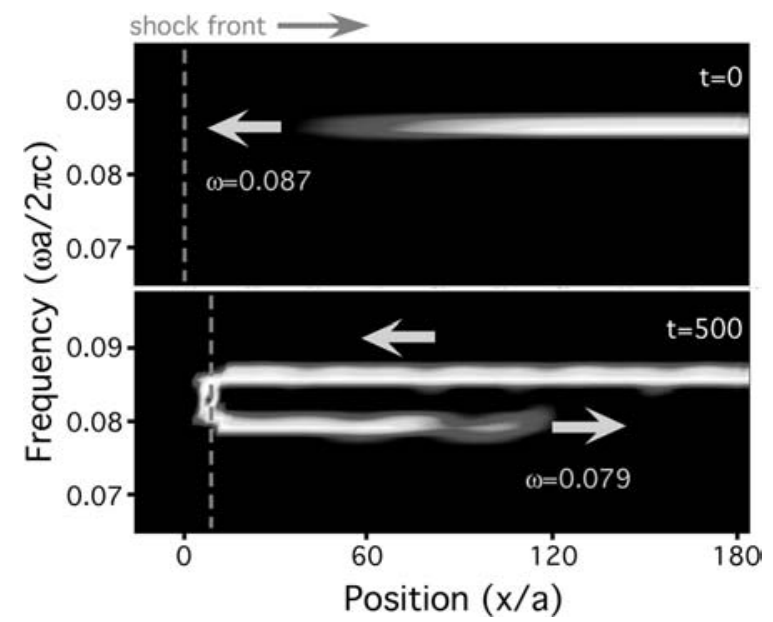

Figure 3. Reverse Doppler effect. Two moments in time during a computer simulation of a pulse of light reflecting from a time-dependent dielectric similar to Figure 1. The shock front is moving to the right and its location is approximately indicated by the dotted line. Light incident from the right receives a negative, i.e. reversed, Doppler shift upon reflection from the shock wave. Time is given in units of $a / c$.

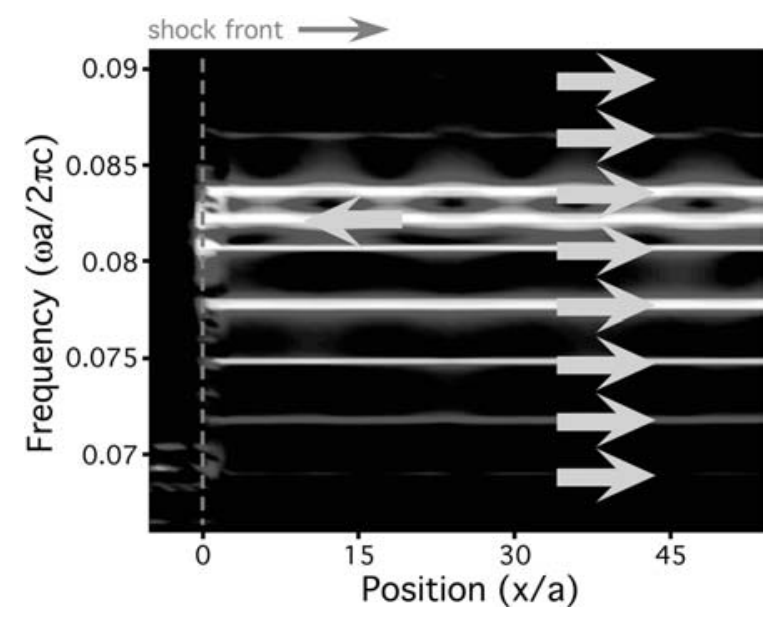

Figure 4. Computer simulation of a pulse of light reflecting from a dielectric similar to Figure 1, but with a sharper shock front than in Figure 3 (by a factor of 20). The shock front is moving to the right and its location is approximately indicated by the dotted line. Light incident from the right is reflected in multiple equally-spaced frequencies due to the relatively sharp shock front.

shift. Figure 5 illustrates how the the usual Doppler effect arises for radiation reflecting from an object that is in motion in some reference frame. As long as there is some moving reference frame in which the object in motion appears to be stationary, the usual Doppler shift arises.

However, if there is no reference frame where the moving object is stationary, the usual Doppler analysis of Figure 5 does not apply. This is the case relevant for the shocked photonic crystal, as illustrated in Figure 6 which shows the dielectric of Figure 1 in a reference frame moving at the shock speed. The dielectric is clearly not time-independent in this reference frame. This observation suggests that the usual 


\section{stationary reference frame}

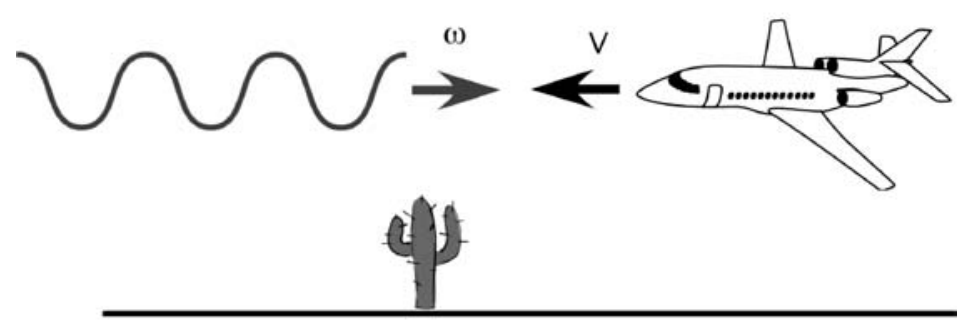

jet reference frame

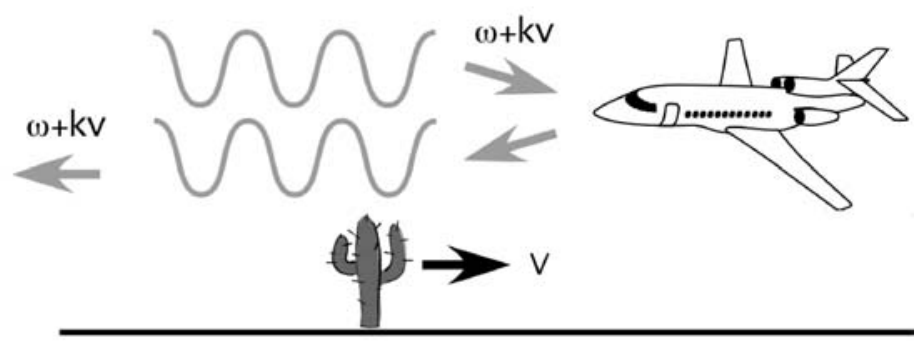

stationary reference frame

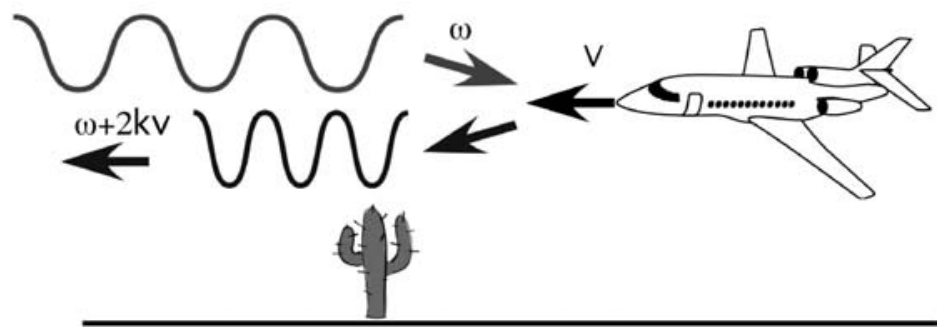

Figure 5. Depiction of the way in which the usual Doppler shift arises. In the top frame, radiation is incident from the left toward a moving object (a jet). In the middle frame, transformation into a reference frame where the jet is at rest yields a shift in the incident radiation frequency. The radiation reflects with no frequency shift in this reference frame. In the bottom frame, transformation back to the original reference frame yields a further shift in the frequency of the reflected light. The usual Doppler shift arises when there is a reference frame where the reflecting object is fixed.

Doppler shift should not necessarily be observed, but does not provide insight into what will actually be observed.

A simple heuristic to understand the reversed Doppler effect is presented in Figure 7. This schematic represents the lower bandgap edge of the photonic crystal as a series of local resonators. Near the bandgap edge, light interacts strongly with the crystal lattice leading to narrower transmission resonances (for a finite-sized crystal) and slower propagation speeds in an unshocked photonic crystal.

As the shock wave propagates to the right in Figure 7, the lower frequency edge of the bandgap is lowered (top panel). This has the effect of moving locally resonant modes down in frequency. Radiation incident from the right (bottom panel) couples into these modes and is moved down in frequency before escaping to the right. Since 


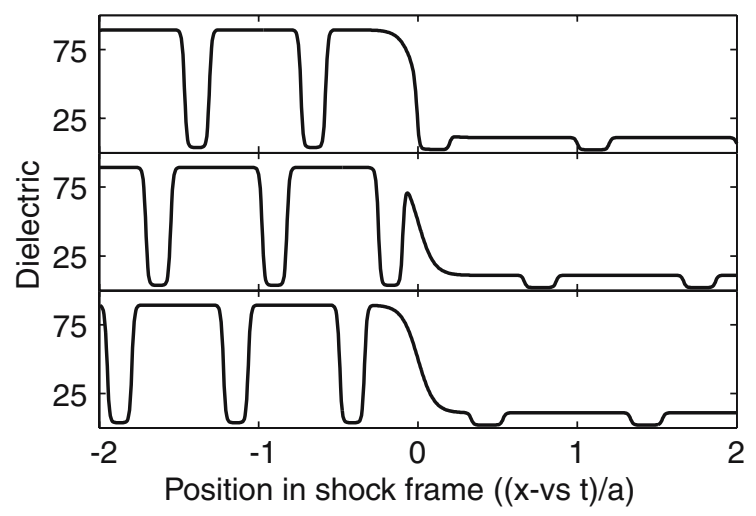

Figure 6. Three time snapshots of the shocked photonic crystal dielectric profile of Figure 1 in a reference frame moving at the shock speed (time increases from the top panel to the bottom panel). This figure shows that the dielectric is time-dependent even in the moving reference frame of the shock, rendering the usual Doppler shift analysis inapplicable. The shock front is located at position 0 in each of the three instances.
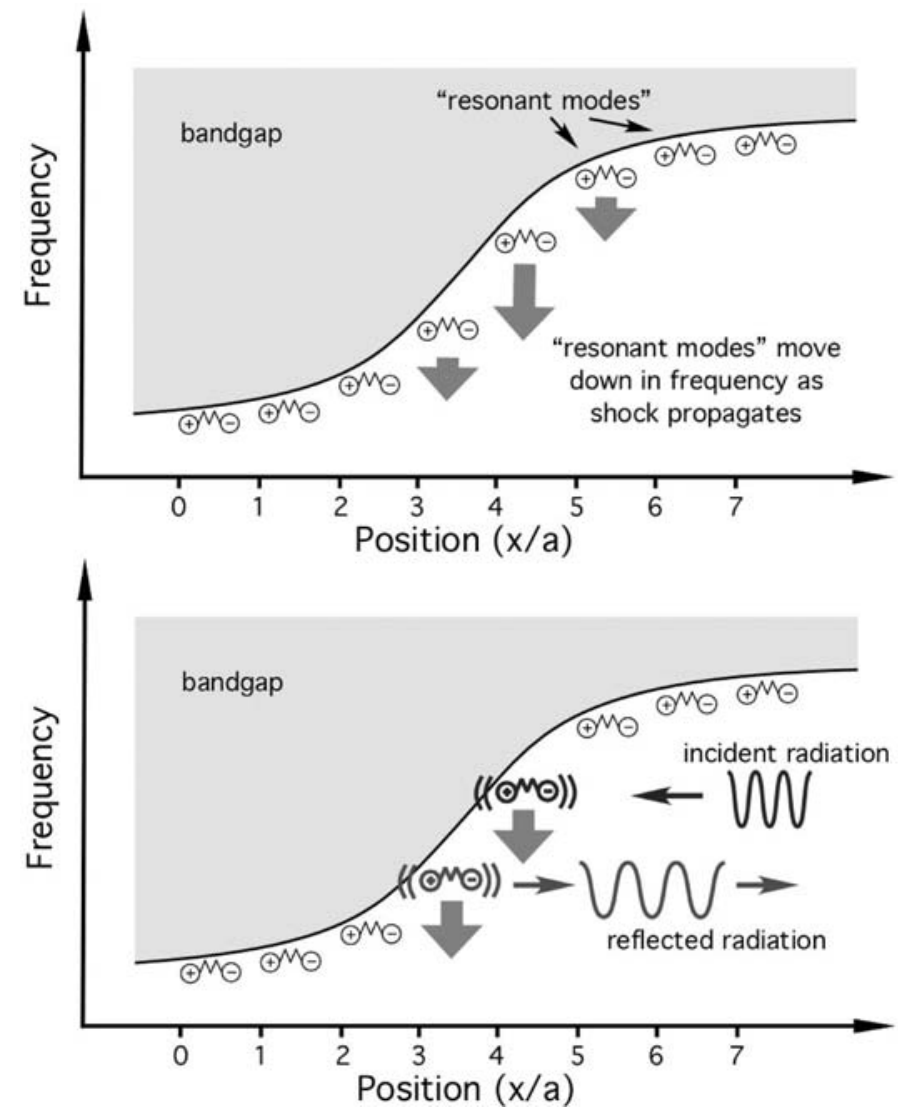

Figure 7. Depicted is a schematic illustrating a reversed Doppler shift when light reflects from a shock wave propagating to the right through a photonic crystal. As the shock propagates, the lower frequency edge of the bandgap is lowered (top panel). This has the effect of moving locally resonant modes down in frequency. Radiation incident from the right (bottom panel) couples into these modes and is moved down in frequency before escaping to the right. Since the shock wave is propagating to the right, a frequency decrease in the radiation is opposite of the usual Doppler shift. 
the shock wave is propagating to the right, a frequency decrease in the radiation is opposite of the usual Doppler shift. The computer simulation in Figure 3 shows light being continuously shifted down in frequency in this fashion at the shock front. This is indicated by the continuous range of frequencies existing between the incident frequency and the reflected frequency.

The reversed Doppler effect can be understood quantitatively in terms of a simple analytical theory. We focus on a scenario where the incident light is at a frequency that falls within the gap of the post-shock, compressed crystal. We choose the incident light frequency to be far below the bandgap of the pre-shock crystal, where it may be described by plane waves. An effective model of the shock front is a mirror with a space-dependent $E$ field reflection coefficient, $R(x)$, where $x$ is the mirror position. $R$ has the property that $|R(x)|=1$ since the incident light reflects from the bandgap of the post-shock crystal. In general, $R$ has some frequency dependence but we will consider the bandwidth of the incident light sufficiently small to neglect it. If the shock front is stationary, the condition on the electric field at the shock front in terms of incident and reflected light is,

$$
\sum_{j} E_{j}^{\prime} e^{-i\left(\omega_{j}^{\prime} t-k_{j}^{\prime} x\right)}=R(x) E_{0} e^{-i\left(\omega_{0} t+k_{0} x\right)},
$$

where $k_{0}$ and $k_{j}^{\prime}$ correspond to the respective incident and reflected wave vectors in the uniform medium and $E_{0}$ and $E_{j}^{\prime}$ are constants. The reflection coefficient $R(x)$ can be written $R(x)=\sum_{G} \beta_{G} e^{-i G x}$ which is the most general form with the property that $R(x)$ is periodic in the pre-shock lattice, $R(x)=R(x+a)$. The reciporical lattice vectors $G$ are $2 \pi q / a$ where $q$ is an integer. This substitution and letting $x \rightarrow x_{0}+v_{\mathrm{s}} t$, where $v_{\mathrm{s}}$ is the shock speed, yields,

$$
\sum_{j} E_{j}^{\prime} e^{-i\left(\omega_{j}^{\prime} t-k_{j}^{\prime}\left(x_{0}+v_{\mathrm{s}} t\right)\right)}=\sum_{G} \beta_{G} e^{-i G\left(x_{0}+v_{\mathrm{s}} t\right)} E_{0} e^{-i\left(\omega_{0} t+k_{0}\left(x_{0}+v_{\mathrm{s}} t\right)\right)} .
$$

The frequency shifts required by the time-dependence of Eq. (6) are,

$$
\omega_{G}^{\prime}-\omega_{0}=\left(k_{0}+k_{G}^{\prime}+G\right) v_{\mathrm{s}} .
$$

The reflected light has frequency components $\omega_{G}^{\prime}$ that differ from the usual Doppler shift, $\left(k_{0}+k_{0}^{\prime}\right) v_{\mathrm{s}}$ (or approximately $2 k_{0 \mathrm{vs}}$ in the non-relativistic limit), by the amount $G v_{\mathrm{s}}$. The amplitude of each of the reflected components is,

$$
\left|E_{G}^{\prime}\right|=\left|\beta_{G}\right|\left|E_{0}\right| \text {. }
$$

When $R(x)$ pertains to the $\ell$ th bandgap, it can be shown that $\left|\beta_{\frac{-2 \pi \ell}{a}}\right|>\left|\beta_{\frac{-2 \pi q}{a}}\right|$ for all $q \neq \ell$. As the shock front is broadened, $R(x) \rightarrow \beta_{\frac{-2 \pi}{a}} e^{\frac{i 2 \pi x}{a}}$ for light reflecting from the first bandgap. These observations are most easily made by considering the adiabatic evolution of the allowed electromagnetic modes as discussed in previous work [14] and to be discussed later in Figure 8. The position dependence of $R(x)$ in this limit can be intuitively understood in the scenario of Figure 7 by considering that the phase of the reflection coefficient should vary as locally resonant modes (modes with low group velocities in this case) move down in frequency through the incident radiation frequency. The phase of the reflected light for a given shock front position 

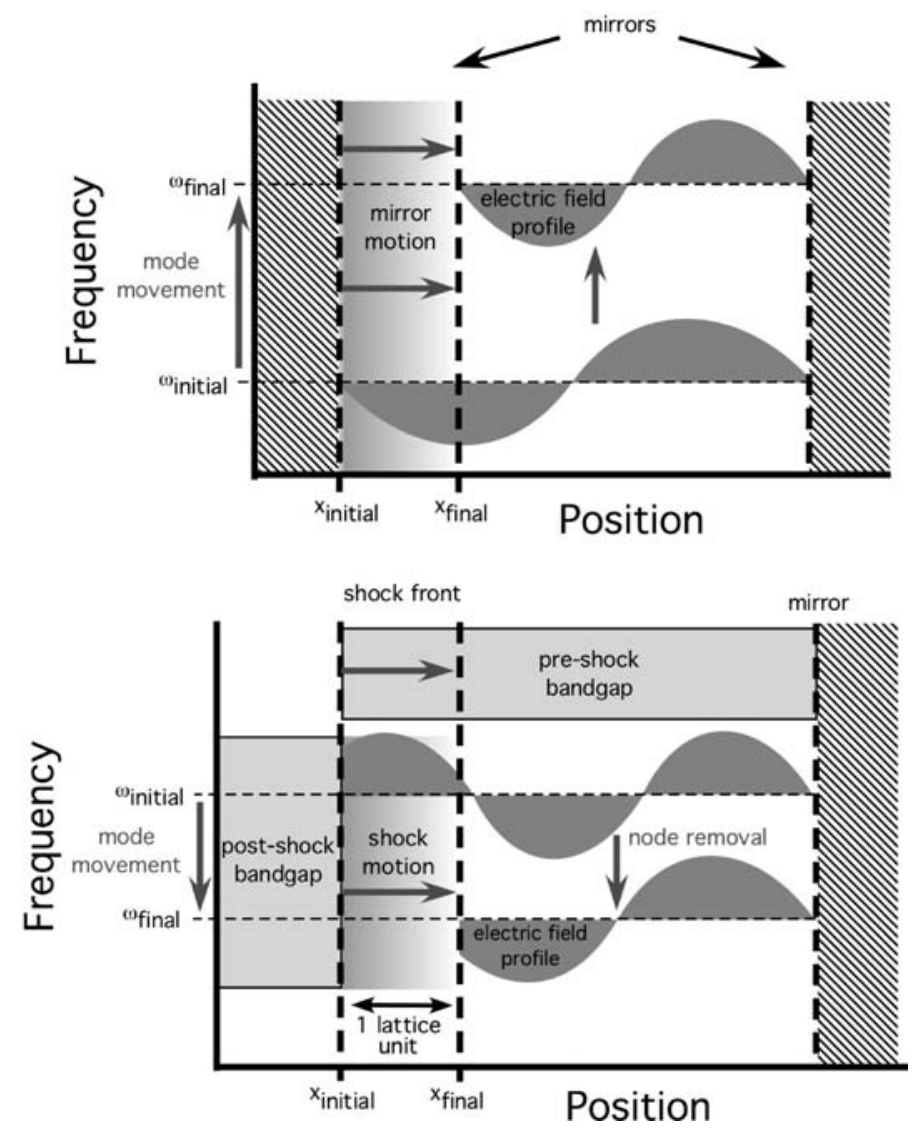

Figure 8. Schematic frequency as a function of position for the normal Doppler shift from a moving metallic mirror (top) and the reversed Doppler shift in a photonic crystal (bottom). Radiation of frequency $\omega_{\text {initial }}$ is confined between a fixed mirror on the right and a moving mirror (top) or shock front (bottom) on the left side. In the top panel, as the left mirror slowly (adiabatically) moves to the right, the number of nodes of the radiation is preserved, giving rise to a frequency increasing effect. A Doppler shift occurs upon each reflection of the radiation from the moving mirror. In this case, the Doppler shift is positive which is the usual Doppler shift. In the photonic crystal in the bottom panel, the lower frequency edge of the lowest frequency bandgap in front of the shock is decreased as the shock propagates. As the shock propagates slowly (adiabatically) to the right through one lattice unit, a node is removed from the field profile by the shift of the reflection phase of the shock front through $2 \pi$. The removal of a node results in a frequency decrease despite the decrease in cavity length, providing an inverse Doppler shift.

is dominated by the frequencies of the locally resonant modes that are close in frequency to the incident radiation frequency.

The reverse Doppler shift scenario in Figure 3 corresponds to this case where the only dominant component of $R(x)=\sum_{G} \beta_{G} e^{-i G x}$ is the one corresponding to $G=$ $-2 \pi / a$. Other frequency components of $R(x)$ are suppressed by the relatively broad shock front width in this case. Eqs. (7) and (8) indicate that the reflected light should have a single frequency with a negative shift if $v_{\mathrm{s}}>0, k_{0}>0$, and $\left|2 k_{0}\right|<2 \pi / a$, which is the case in Figure 3. In Figure 4, the relatively sharp shock front gives rise to multiple reflected frequencies separated by $\frac{2 \pi v_{\mathrm{s}}}{a}$. 
Equation (7) predicts that when $2 k_{0}=-G$ and there is only one reflected frequency component, the Doppler shift is zero. Likewise, if $2 k_{0}>-G$, the Doppler shift is positive (normal) but has a magnitude that is smaller than the usual $2 k_{0} v_{\mathrm{s}}$ Doppler shift. Both of these scenarios have been observed in our finite-difference simulations.

We would like to emphasize that it is not possible to observe these anomalous effects by simply translating a photonic crystal through a uniform medium because the reflection coefficient for the photonic crystal in that case is constant in time, as in the case of a metal mirror. The key new physical phenomena presented here result from the fact that the shocked photonic crystal region "grows" into the pre-shocked region giving rise to a time-dependent reflection coefficient. While the material behind the shock moves with some speed other than the shock speed (the so-called particle speed), it is interesting to note that this velocity plays no role in the Doppler shift phenomenon. Only the shock front velocity is relevant.

Figure 8 shows another schematic depiction of the origin of the inverse Doppler effect in this system. First consider the origin of the usual Doppler effect in the system shown in the top panel. Suppose the left mirror moves to the right sufficiently slowly that the electromagnetic mode evolves adiabatically. As the left mirror moves to the right, the nodal structure of the mode is preserved and the frequency is increased due to a decrease in cavity length. A Doppler shift occurs each time the light reflects from the moving mirror. In this case, the Doppler shift has a positive sign, which is the usual Doppler shift.

Now consider the origin of the inverse Doppler effect in a photonic crystal in the bottom panel of Figure 8. As the shock propagates to the right through one lattice unit, a node is removed from the electric field profile by the shift of the reflection phase of the shock front, and the frequency shifts down. The decrease of the cavity length tends to increase the frequency but the removal of a node yields a larger frequency decreasing effect in this particular case. The Doppler shift has a negative sign, which is an inverse Doppler shift. Another way to appreciate this frequency shift is to note that modes must move down in frequency because they all start out in the frequency range below the bandgap in the pre-shock crystal and all end (after the shock has propagated through the entire crystal) in the frequency range below the post-shock bandgap which is lower than the pre-shock bandgap.

In the finite-difference simulations, we have chosen a $10 \mathrm{GPa}$ pressure shock with large shock speeds and large strain dependence of the dielectric due to consideration for computational effort. The effects presented here are just as easily observable over a wide range of shock pressures, realistic shock speeds $(1-10 \mathrm{~km} / \mathrm{sec})$, and realistic values of $\frac{1}{\epsilon} \frac{d \epsilon}{d s}$. Materials are routinely shocked to GPa and higher pressures using lasers and gun-driven projectile impacts. Optical techniques involving the reflection of light from a moving shock front are used as diagnostics in shock experiments [20, 21]. Spectroscopic techniques possess ample resolution to observe the shifts proposed here which can be as small as a normal Doppler shift from an object moving on the order of $100 \mathrm{~m} / \mathrm{s}$ [22]. It is also interesting to note that measurement of the properties of the reflected light in such an experiment allows determination of the shock front thickness as in Figures 3 and 4. This is currently difficult or impossible to accomplish and may represent a new tool for the study of shock waves.

For small shock pressures, the requirement that the light be in the linear dispersion frequency region of the pre-shocked crystal (away from the band edge) can be 
accomplished by using a crystal with a small bandgap, e.g. by using a large layer of a material with large negative $\frac{d \epsilon}{d s}$ and a small layer of another material with a different dielectric. In this case, the condition on $\frac{d \epsilon}{d s}$ for the bandgap to decrease upon compression is $\frac{1}{\epsilon} \frac{d \epsilon}{d s}<-2$. Materials used for acoustic light modulation, like Te, or other high dielectric materials can be used to satisfy this condition. Alternatively, if light is reflected from the rear of the shock front (i.e. the incident light propagates the same direction as the shock), materials with $\frac{1}{\epsilon} \frac{d \epsilon}{d s}>-2$ (i.e. all other materials) can be employed to observe a reversed Doppler shift if the incident light is of a frequency within the bandgap of the pre-shock crystal.

Shock impedance matching between the two bilayers of the photonic crystal is important for the propagation of a steady shock wave. A wide variety of optical materials of varying impedances exist to simultaneously establish good impedance matching and some dielectric contrast, for example Te and LiF. These two materials are not required to possess identical sound speeds because the reflection coefficient $R(x)$ is periodic in time even when the sound speeds differ. Differing bulk moduli between the two materials has little effect on the gap position when one of the bilayers is substantially smaller than the other.

While the emphasis of this section has been on the observation of anomalous Doppler shifts in a shocked photonic crystal, similar shifts can be observed in a variety of time-dependent photonic crystal systems and other periodic electromagnetic systems. The next section describes an observation of the reversed Doppler effect in an electrical transmission line.

\section{Experimental observation of a reversed Doppler effect}

Seddon and Bearpark (SB) have recently observed a reversed Doppler effect in an electrical transmission line [1]. Rather than a photonic crystal, a 1D radio-frequency electrical transmission line comprised of coupled inductor-capacitor resonators was utilized. In our interpretation of this experiment, the transmission line possessed a cut-off frequency above which radiation is reflected, as depicted in Figure 9. The cut-off frequency can be considered analogous to the lower frequency edge of a 1D photonic crystal bandgap. A shock-like wave was generated by utilizing non-linear behavior of the inductors under application of a current pulse sent into the transmission line. As the current pulse propagated through the transmission line (changing the inductances of the inductors as it travelled) the local cut-off frequency was changed as illustrated in Figure 9. GigaHertz frequency radiation was generated and reflected from the shock front, yielding a frequency increase in the reflected radiation. The usual Doppler shift predicts a frequency decrease in this scenario, opposite of what was observed.

Two fundamentally different theoretical explanations have been proposed for the observation of the reversed Doppler effect under the experimental conditions of SB. One of these explanations is due to SB $[1,23]$ and the other is our theory described in the previous sections and in previous work $[15,24]$. These two theories provide quantitatively identical predictions in conditions of the experiment of SB, but provide differing predictions in other experimental regimes. 

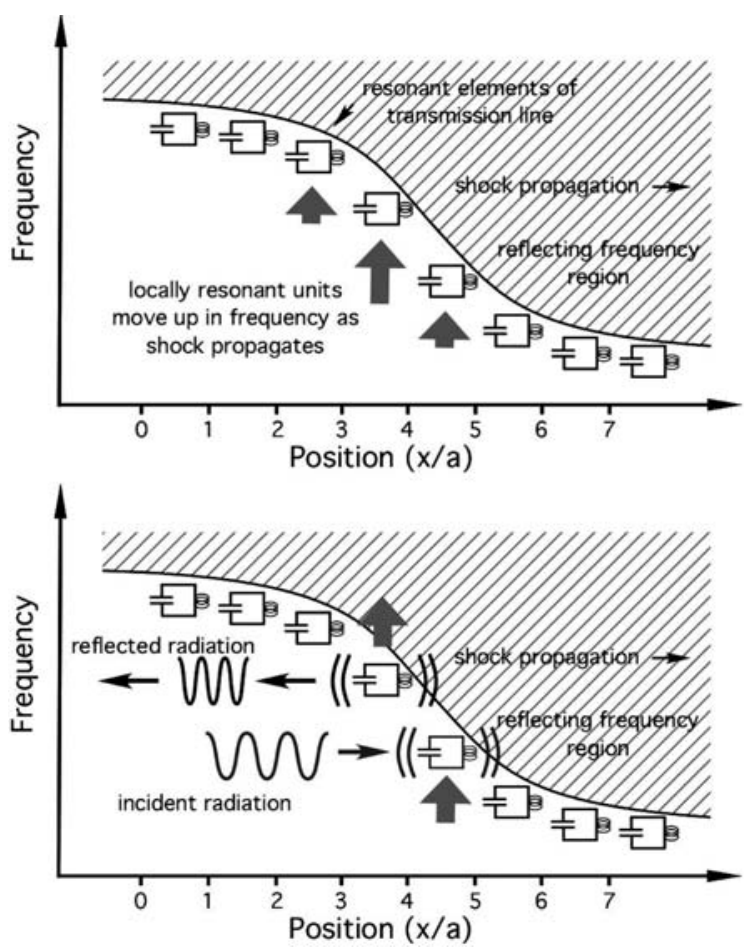

Figure 9. Depicted is a schematic of our interpretation of the reversed Doppler effect observed by Seddon and Bearpark in a transmission line. The shock changes the cut-off frequency of the transmission line as it propagates to the right (top panel). Radiation incident from the left couples into resonant portions of the transmission line and is shifted up in frequency before escaping to the left (bottom panel.)

Our theoretical description of this experiment is schematically illustrated in Figure 9 and is closely related to the schematic description of the reversed Doppler effect in the photonic crystal case illustrated in Figure 7. Radiation incident from the left couples into local resonances that are moved up in frequency by the shock. Radiation escapes from the local resonances to the left with a higher frequency, opposite of that expected for the usual Doppler shift in this case. The anomalous frequency shift results from the unusual reflecting surface that the shock front represents and is independent of the nature of the electromagnetic modes that contain the incident and reflected electromagnetic radiation. The experimentally observed frequency shift quantitatively agrees with Eq. (7) when $G=2 \pi / a$, expected to be the dominant $G$ component of the reflection coefficient $R(x)$ representing the shock wave in this case.

The theoretical description of SB involves the reflection of unusual electromagnetic waves (backward waves with $v_{\text {phase }} v_{\text {group }}<0$ ) from a reflecting shock front with fixed reflection phase shift. The reversed Doppler shift results from the unusual nature of the electromagnetic waves within this description rather than an unusual reflecting surface. This picture is closely related to the effect predicted to occur in negativeindex materials (rather than transmission lines) made by Veselago [16].

While the theory presented in this work and the theory of SB happen to predict the same results in the experimental conditions of SB, differing results are predicted in other regimes of the SB system. For example, our analysis also predicts that 


\section{Evan J. Reed et al.}

multiple frequencies may be reflected from the shock as the shock front thickness is decreased as in Figure 4, while the SB analysis provides no mechanism for more than one frequency to be emitted.

\section{Conclusion}

We have predicted that reversed and anomalous Doppler shifts can occur when light reflects from a shock wave propagating through a photonic crystal. This effect has been experimentally observed by Seddon and Bearpark when a shock-like wave propagated through an electrical transmission line. In addition to shock waves and shocklike waves, we expect that dynamically modifying periodic media while light interacts with the media will make possible new and useful techniques for the manipulation of electromagnetic radiation.

\section{Acknowledgements}

The authors are particularly grateful to Nigel Seddon for many helpful discussions. The authors also thank Neil Holmes, Jerry Forbes, and David Hare for helpful discussions. Evan Reed acknowledges support from the Department of Defense NDSEG fellowship, and the Lawrence Livermore National Laboratory MRI/EMC fellowship. This work was supported in part by the Materials Research Science and Engineering Center program of the National Science Foundation under Grant No. DMR-9400334. This work was performed in part under the auspices of the U.S. Department of Energy by University of California, Lawrence Livermore National Laboratory under Contract W-7405-Eng-48.

\section{References}

1. Seddon, N. and Bearpark, T., Science, 302 (2003) 1537.

2. Yablonovich, E., Phys. Rev. Lett., 58 (1987) 1059.

3. John, S., Phys. Rev. Lett., 58 (1987) 2486.

4. Joannopoulos, J.D., Meade, R.D. and Winn, J.N., Photonic Crystals, Princeton University Press, Princeton, NJ, 1995.

5. Taflove, A. and Hagness, S.C., Computational Electrodynamics: The Finite-difference Timedomain Method, Artech House, Norwood, MA, 2000.

6. Shimoda, Y., Ozaki, M. and Yoshino, K., Appl. Phys. Lett., 79 (2001) 3627.

7. Leonard, S.W., van Driel, H.M., Schilling, J. and Wehrspohn, R.B., Phys. Rev. B, 66 (2002) 161102.

8. Hu, X., Zhang, Q., Liu, Y., Cheng, B. and Zhang, D., App. phys. Lett., 83 (2003) 2518.

9. Skorobogatiy, M. and Joannopoulos, J.D., Phys. Rev. B, 61 (2000) 5293.

10. Skorobogatiy, M. and Joannopoulos, J.D., Phys. Rev. B, 61 (2000) 15554.

11. Yanik, M.F., Suh, W., Wang, Z. and Fan, S.H., Phys. Rev. Lett., 93 (2004) 233903.

12. Yanik, M.F. and Fan, S.H., Phys. Rev. Lett., 93 (2004) 173903.

13. Yanik, M.F. and Fan, S.H., Phys. Rev. Lett., 92 (2004) 083901.

14. Reed, E.J., Soljačić, M. and Joannopoulos, J.D., Phys. Rev. Lett., 90 (2003) 203904.

15. Reed, E.J., Soljačić, M. and Joannopoulos, J.D., Phys. Rev. Lett., 91 (2003) 133901.

16. Veselago, V.G., Sov. Phys. USPEKHI, 10 (1968) 509.

17. Belyantsev, A.M. and Kozyrev, A.B., Tech. Phys., 47 (2002) 1477.

18. Einat, M. and Jerby, E., Phys. Rev. E, 56 (1997) 5996.

19. Dixon, R.W., J. Appl. Phys., 38 (1968) 5149. 
20. Gustavsen, R. and Gupta, Y.M., J. Appl. Phys., 69 (1991) 918.

21. Celliers, P.M., Collins, G.W., Silva, L.B.D., Gold, D.M., Cauble, R., Wallace, R.J., Foord, M.E. and Hammel, B.A., Phys. Rev. Lett., 84 (2000) 5564.

22. Holmes, N.C., Rev. Sci. Instrum., 64 (1993) 357.

23. Seddon, N., and Bearpark, T., Science, 304 (2004) 778

24. Reed, E.J., Soljačić, M., Ibanescu, M. and Joannopoulos, J.D., Science, 304 (2002) 778. 